\title{
The LIVE project: A Protocol for Rapid Living Reviews of HIV Implementation Research
} \author{
9868 \\ Nathan Ford \\ World Health Organization

\section{Baral Stefan} \\ Johns Hopkins University \\ Sheree Schwartz \\ John Hopkins University \\ Elvin H Geng \\ Washington University in Saint Louis
}

Ingrid Eshun-Wilson ( $\nabla$ i.eshun-wilsonova@wustl.edu )

Stellenbosch University Faculty of Medicine and Health Sciences https://orcid.org/0000-0002-4049-

\section{Protocol}

Keywords: HIV, implemention science, meta-analysis, living review, HIV cascade, rapid review

Posted Date: April 30th, 2020

DOl: https://doi.org/10.21203/rs.3.rs-24965/v1

License: (c) (i) This work is licensed under a Creative Commons Attribution 4.0 International License.

Read Full License 


\section{Abstract}

Background: HIV implemention research evolves rapidly and is often complex and poorly characterized, making synthesis of data on HIV implementation strategies inherently difficult. This is further compromised by prolonged data abstraction processes, restrictions on study design and delays in publication of review findings, at times resulting in outdated and irrelevant systematic reviews. The LIVE project aims to overcome some of these challenges by conducting rapid living systematic reviews and meta-analyses.

Methods: The LIVE team will generate a series of systematic reviews exploring implementation strategies targeted at improving HIV cascade outcomes. We will search Embase and Medline databases for studies conducted after 2004, using predefined search terms to identify studies conducted in any age group or setting, and using implementation strategies - targeting policy makers, society, health organizations, health workers, and patients and their families - to prevent HIV, increase HIV testing, increase ART linkage and retention, and improve viral suppression rates and survival. Randomized controlled trials and observational studies will be identified through optimized searches conducted six-monthly and features of the implementation strategies and methodological quality will be assessed using recognized tools. Review teams will publish living systematic review protocols on PROSPERO. Review results will be presented on a publically facing dashboard, with review updates conducted on a six monthly basis, and publication in peer reviewed journals supporting living review processes.

Discussion: Living reviews based on both pragmatic and explanatory HIV implementation research and supported by an ongoing data abstraction process will generate rapid reviews in the HIV field which are current, relevant and maximally useful to patients, health workers and policy makers.

\section{Background}

Systematic reviews addressing HIV implementation research questions become outdated rapidly. As HIV prevention strategies, testing methods and treatments become increasingly effective, current primary HIV research and evidence synthesis questions are refocusing on how best to implement effective interventions to ensure long-term sustained engagement in HIV care (1). This continuous emergence of new implementation research means that traditional 'static' systematic reviews, that may take months or years to produce, quickly become obsolete $(2,3)$. And, with each new guideline development cycle, new review teams, searches, protocols and reviews are undertaken, resulting in substantial duplication of efforts, delays in the generation of synthesized evidence and inability for guideline developers to quickly update recommendations.

Living and rapid review methods have been developed in recent years to address these inefficiencies and increase the utility of review evidence, these methods have however been infrequently applied to HIV implementation research. Living methods offer an approach for review updating, where new evidence is incorporated into a review as it emerges, generating a continual updating process which maintains the 
relevance of synthesized findings and builds on previous work. Living reviews require an explicitly stated commitment to a predetermined frequency of searches and review updating (4). Rapid reviews aim to accelerate the review process through the elimination or attenuation of some systematic review requirements. Rapid processes include conducting searches in fewer databases, applying language or publication year restrictions, limiting gray literature searches, applying data mining processes, and altering duplicate screening, data extraction and quality appraisal processes $(5,6)$. Findings from rapid reviews appear to have comparable results to more rigorous systematic reviews $(5,7)$ and are being conducted with increased frequency to respond to policy-making needs $(8,9)$.

The LIVE project aims to generate a series of methodologically robust rapid and living reviews evaluating the effects of HIV intervention implementation strategies on HIV cascade outcomes through an ongoing process of data abstraction and frequent review updates, to produce valid and relevant synthesized evidence that contributes to the public health response to HIV. This protocol outlines the LIVE project review processes.

\section{Objective}

To produce living rapid reviews and meta-analyses of the effectiveness of HIV implementation strategies on HIV cascade outcomes, to support HIV policy making processes and identify research gaps.

\section{Review Methods}

This protocol was designed according to Preferred Reporting Items for Systematic Reviews and MetaAnalyses Protocols (PRISMA-P), living review guidelines (4) and WHO and Cochrane rapid review guidelines $(6,9)$.

Identification of review questions:

Revelant HIV implementation science questions will be developed in consultation with HIV guideline development groups. This will include questions regarding effectiveness and efficacy of HIV implementation strategies. Individual review protocols will be published on PROSPERO the international prospective register of systematic reviews (https://www.crd.york.ac.uk/prospero/).

Eligibility criteria:

Studies eligible for inclusion in living rapid reviews include those conducted in any population group or age category from any setting. Randomized controlled trails (RCTs), cohort studies (with or without a comparison arm), cross-sectional studies and natural experiments are eligible for inclusion. Studies must evaluate implementation interventions (interventions aimed at implementing a change to the way HIV care and preventions strategies are delivered or modify patient behavior to improve outcomes) and report on at least one HIV cascade outcome (diagnosis, testing, linkage to ART, retention on ART, ART adherence, 
viral suppression, and survival) (Table 1). Eligible studies will be restricted to English language publications.

Table 1: Eligibility criteria for inclusion in LIVE rapid living reviews

\begin{tabular}{|l|l|}
\hline Population & All settings ; all ages \\
\hline Intervention & $\begin{array}{l}\text { Interventions aimed at: (1) implementing a change to the way HIV care and preventions strategies are delivered or (2) modify } \\
\text { patient behavior }\end{array}$ \\
\hline Comparison & Other intervention, standard of care, or no comparison \\
\hline Outcome & HIV incidence, HIV testing uptake, ART initiation, ART adherence, viral suppression, mortality \\
\hline
\end{tabular}

Database searches:

A search specialist will conduct searches of a minimum of two databases Medline and Embase, and will include CINAHL and other databases depending on the considered added value for the specified review question. Search outputs will be refined through an iterative process of cross-checking against known studies in the field and once finalized, automated searches, running at a pre-determined frequency (6 monthly), will generate updated lists of studies for screening and eligibility assessment and abstraction. Searches will include studies published between 2004 to present day, but may be be restricted to more recent studies depending on the specified review question. Search strategies will be re-evaluated and revised on a 12 monthly basis to ensure search terms reflect the current HIV implementation research terminology and that relevant studies are captured.

Gray literature searches:

At minimum the International AIDS Society conferences ( https://www.iasociety.org/Conferences) and Conference on Retroviruses and Opportunistic Infections (https://www.croiconference.org/) conference abstracts will be searched for the previous two years. Additional conference searches will depend on their relevance to review questions. Decisions regarding the searching of clinical trial registries such as Clinical trials.gov and WHO ICRTP Clinical trial registries will depend on the specific review, if few studies are known in the field, exploring trial regristries may be necessary.

Screening:

Several team members may be involved in screening processes. Abstract and full-text screening will be conducted using Covidence software (10). For abstract screening, two team members will screen the first $20 \%$ of abstracts with conflict resolution, once approaches to screening are calibrated, ongoing abstract screening will be conducted by one team member. Full-text screening will be conducted by one team member and excluded full-texts will be screened by a second. Conferences and clinical trial registry searches will be conducted by one team member with confirmation of eligibility of included abstracts by a second.

Data abstraction: 
Study data will be abstracted into the LIVE database hosted on the Airtable platform (a relational database designed to be easily modified by end users and widely used commercially (11). Basic descriptive information will be extracted from each study (including details on publication, study design, setting, context and demographic characteristics) and additional data regarding the critical characteristics and components of implementation strategies are recorded using existing frameworks for evaluating implementation strategies to guide content extraction (12) as well as characterization of intervention mechanisms (13) (Table 2). Extracted study outcomes will include numerators and denominators, as well as adjusted and unadjusted effect estimates. Data abstraction and methological quality assessments will be conducted by one team member and reviewed by a second.

Table 2: Tools used to assess study quality and characterize intervention strategies for living rapid reviews

\begin{tabular}{|l|l|}
\hline Assessment Tool & Purpose \\
\hline Cochrane Risk of bias tool version 1 or $2(14,15)$ & Methodological quality of randomized controlled trials \\
\hline Newcastle Ottawa scale (16) & Assess methodological quality of cohort and cross-sectionals studies \\
\hline $\begin{array}{l}\text { Standards for Reporting Implementation Studies } \\
\text { (StaRI) (17) }\end{array}$ & Assess reporting of implementation science studies \\
\hline PRECIS-2 tool (13) & Evaluate explanatory vs pragmatic approaches of studies \\
\hline
\end{tabular}

\section{Analyses}

We will characterize individual study populations, implementation interventions, comparisons and HIV cascade outcomes and other outcomes relevant to the review questions including harms and unintended consequences. If there is sufficient quantitative data, these data will be meta-analyzed, in R, Stata or SAS programmes and depending on the type of data available for analysis (e.g., continuous, binary, incidence, adjusted effect estimates, single means or proportions) pooled results and forest plots for random effects will be generated using Mantel-Hansel, Peto, generalized linear models, and generic inverse variance (18). We will review inconsistency qualitatively, and quantitatively using $I^{2}$, Kendall's tau statistics. Decisions regarding the appropriateness of pooling data, sub-grouping and sensitivity analyses will be conducted by study teams. In addition where multiple strategies are presented, network meta-analyses may be conducted and will follow guidelines for conduct and reporting of network metaanalyses. The frequentist or Bayesian network meta-analysis (NMA) approaches will be used to generate networks, evaluate inconsistency, rank interventions. Although the inherent nature of implementation strategies may in some cases violate the assumption of transitivity due to variability in context and intervention heterogeneity - in terms of design and fidelity to intervention delivery - this analytic technique allows for the comparison of multiple interventions which have not been compared directly due to public health urgency and resource constraints (19). Where data are insufficient for meta-analysis we will summarize data narratively. The overall confidence in the review findings will be evaluated using recognized methodologies for rating evidence certainty such as the GRADE system (20).

\section{Living Processes}


Once a review is completed, a continuous - living - process will be adopted to keep the review findings up to date and presented on a publically facing web-based LIVE dashboard $(4,21)$. A comprehensive systematic search will be conducted every six months. If no new studies are detected, we will update review records on the LIVE dashboard with the most recent search date and specify that no new relevant studies have been identified. If enough new studies are identified at the six month timepoint and no changes are required to the original review methods, the new evidence will be immediately incorporated into the review and meta-analyses, search details will be updated, results will be adjusted on the LIVE dashboard. If there are significant changes to the original results and conclusions of the primary publication, we will discuss a new publication plan with the publishing journal. If the update requires changes to the original review methodology, we will update the published protocol on PROSPERO. With each six month cycle the LIVE team will additionally consider whether reviews should be retired, considerations for retiring reviews will be based on whether the review question remains relevant in the field, as the importance of specific research questions may have changed over time (22).

\section{Discussion}

The LIVE project will generate uptodate and relevant rapid living reviews that will characterize HIV implementation strategies, detailing the mechanisms of - where, how and by whom evidence based interventions are delivered, to determine how these and other strategy features impact HIV incidence, diagnosis, linkage and retention in HIV care. Incorporation of a broad range of study designs including both randomized controlled trials and observational studies will facilitate exploration of both the efficacy of intervention strategies and how strategies perform under real-world conditions.

HIV research is now at a point where, accurate rapid diagnostic techniques are available and effective interventions exist - for both HIV prevention and treatment - but mechanisms for reaching and engaging all who need to be diagnosed and treated, are incompletely identified. We need primary research which is focused on implementation (23), and evidence synthesis methods which accommodate complexity, recognizing that heterogeneity is an inherent feature of the current HIV response, and essential (24). Heterogeneity does not preclude evidence synthesis, but rather highlights the need to develop approaches to accommodate varied study designs and implementation strategies and still draw conclusions from the evidence. To do this LIVE reviews will - in addition to characterizing intervention complexity and conducting subgroup analyses - utilize analytic methods such as network meta-analysis to explore and synthesize heterogenous implementation strategies.

Employing rapid and living processes will make LIVE reviews ideal for incorporation into 'living guidelines' - a dynamic guideline development process that, instead of conducting mechanistic guideline updates with a predetermined frequency, uses the results of continuous literature surveillance, rapid updating of prioritized reviews and frequent virtual consultations with guideline panels, to create a continuous guideline development and revision process, with the goal of ensuring that policy makers and health workers can make uptodate evidence based public health decisions (25-27). Living guidelines have been developed for reproductive health research but to date HIV guidelines have not adopted this approach. 
The LIVE project is well positioned to facilitate the development of living guidelines for implementation research in the HIV field.

We believe that if successful, this project could ultimately enhance the use of science to improve health across disease conditions and therefore broadly impact the public. The idea that research should be widely relevant to routine health care systems is nearly axiomatic, but the ways in which the scientific and public health communities produce, synthesize, and use data is not yet optimal. Substantial delays in the translation of both primary and implementation research findings to policy makers and health-workers can prevent early and effective adoption of new strategies for improving health - the evidence-practice gap $(28,29)$. Living and rapid reviews can play a critical role in bridging this gap by synthesizing complex and varied research findings into more digestible forms for policy-makers and consumers (3).

The LIVE project will generate relevant rapid living reviews of HIV implementation research which advance implementation science evidence synthesis and accelerate the development of recommendations for HIV care.

\section{Abbreviations}

HIV: Human Immunodeficiency Virus; LMIC: Low and middle income country; NMA: Network metaanalysis

\section{Declarations}

Ethics approval and consent to participate: not applicable

Consent for publication: not applicable

Availability of data and materials: not applicable - protocol

Competing interests: Authors have no competing interests

Funding: NIH: K24 Al134413; Bill and Melinda Gates Foundation: OPP1215984; Viiv Healthcare. The funders had no role in conceptualizing or developing this protocol.

Authors' contributions: All authors contributed to the conceptualization of this protocol and reviewed and approved the final manuscript.

Acknowledgements: Jeanna Wallenta, Chris Kemp

\section{References}

1. Geng EH, Holmes CB, Moshabela M, Sikazwe I, Petersen ML. Personalized public health: An implementation research agenda for the HIV response and beyond. PLoS medicine. 
2020;16(12):e1003020.

2. Eshun-Wilson I, Jaffer S, Smith R, Johnson S, Hine P, Mateo A, et al. Maintaining relevance in HIV systematic reviews: an evaluation of Cochrane reviews. Systematic reviews. 2019;8(1):46.

3. Elliott JH, Turner T, Clavisi O, Thomas J, Higgins JPT, Mavergames C, et al. Living Systematic Reviews: An Emerging Opportunity to Narrow the Evidence-Practice Gap. PLoS medicine. 2014;11(2):e1001603.

4. Elliott JH, Synnot A, Turner T, Simmonds M, Akl EA, McDonald S, et al. Living systematic review: 1. Introduction-the why, what, when, and how. J Clin Epidemiol. 2017;91:23-30.

5. Tricco AC, Antony J, Zarin W, Strifler L, Ghassemi M, Ivory J, et al. A scoping review of rapid review methods. BMC medicine. 2015;13(1):224.

6. Cochrane. Cochrane Rapid Reviews. Interim Guidance from the Cochrane Rapid Reviews Methods Group 2020 [Available from: https://methods.cochrane.org/rapidreviews/cochrane-rr-methods.

7. Haby MM, Chapman E, Clark R, Barreto J, Reveiz L, Lavis JN. What are the best methodologies for rapid reviews of the research evidence for evidence-informed decision making in health policy and practice: a rapid review. Health research policy and systems. 2016;14(1):83-.

8. Kuchukhidze S, Long L, Pascoe S, Huber A, Nichols B, Fox M, et al. Differentiated models of service delivery (DSD) for antiretroviral treatment of HIV in sub-Saharan Africa: A review of the gray literature as of June 2019. AMBIT Project Report Number 03. Boston: Boston University and HE2RO, 2019.; 2019.

9. Tricco AC, Langlois EV, Straus SE, editors. Rapid reviews to strengthen health policy and systems: a practical guide. Geneva: World Health Organization. 2017.

10. Covidence. Covidence systematic review software, Veritas Health Innovation. Melbourne, Australia2016.

11. Garcia de Cortazar AR, Cabrera Leon A, Hernan Garcia M, Jimenez Nunez JM. Attitudes of adolescent Spanish Roma toward noninjection drug use and risky sexual behavior. Qualitative health research. 2009;19(5):605-20.

12. Proctor EK, Powell BJ, McMillen JC. Implementation strategies: recommendations for specifying and reporting. Implement Sci. 2013;8:139.

13. Loudon K, Treweek S, Sullivan F, Donnan P, Thorpe KE, Zwarenstein M. The PRECIS-2 tool: designing trials that are fit for purpose. BMJ : British Medical Journal. 2015;350:h2147.

14. Higgins JP, Altman DG, Gotzsche PC, Juni P, Moher D, Oxman AD, et al. The Cochrane Collaboration's tool for assessing risk of bias in randomised trials. BMJ. 2011;343:d5928.

15. Group RD. RoB 2: A revised Cochrane risk-of-bias tool for randomized trials 2019 [Available from: https://methods.cochrane.org/bias/resources/rob-2-revised-cochrane-risk-bias-tool-randomizedtrials.

16. Wells GA, Shea B, O'Connell D, Peterson J, Welch V, Losos M, et al. The Newcastle-Ottawa Scale (NOS) for assessing the quality if nonrandomized studies in meta-analyses [Available from: 
http://www.ohri.ca/programs/clinical_epidemiology/oxford.asp.

17. Pinnock H, Barwick M, Carpenter CR, Eldridge S, Grandes G, Griffiths CJ, et al. Standards for Reporting Implementation Studies (StaRI) Statement. BMJ. 2017;356:i6795.

18. R Core Team. R: A language and environment for statistical computing. R Foundation for Statistical Computing, Vienna, Austria. 2013 [Available from: URL http://www.R-project.org/.

19. Geng EH, Petersen ML. Network meta-analyses: powerful but not without perils. The lancet HIV. 2014;1(3):e95-6.

20. Guyatt G, Oxman AD, Akl EA, Kunz R, Vist G, Brozek J, et al. GRADE guidelines: 1. Introduction-GRADE evidence profiles and summary of findings tables. J Clin Epidemiol. 2011;64(4):383-94.

21. Counotte MJ, Kim CR, Wang J, Bernstein K, Deal CD, Broutet NJN, et al. Sexual transmission of Zika virus and other flaviviruses: A living systematic review. PLoS medicine. 2018;15(7):e1002611.

22. Garner P, Hopewell S, Chandler J, MacLehose H, Akl EA, Beyene J, et al. When and how to update systematic reviews: consensus and checklist. BMJ. 2016;354:i3507.

23. Ford N, Geng E, Ellman T, Orrell C, Ehrenkranz P, Sikazwe I, et al. Emerging priorities for HIV service delivery. PLoS medicine. 2020;17(2):e1003028.

24. Geng EH, Holmes CB, Moshabela M, Sikazwe I, Petersen ML. Personalized public health: An implementation research agenda for the HIV response and beyond. PLoS medicine. 2019;16(12):e1003020.

25. Vogel JP, Dowswell T, Lewin S, Bonet M, Hampson L, Kellie F, et al. Developing and applying a \&\#039;living guidelines\&\#039; approach to WHO recommendations on maternal and perinatal health. BMJ global health. 2019;4(4):e001683.

26. WHO. World Health Organization. Keeping global recommendations up-to-date: A 'living guidelines' approach to maternal and perinatal health. 2020 [Available from: https://www.who.int/reproductivehealth/mph-living-guidelines/en/.

27. Akl EA, Meerpohl JJ, Elliott J, Kahale LA, Schunemann HJ, Living Systematic Review N. Living systematic reviews: 4. Living guideline recommendations. J Clin Epidemiol. 2017;91:47-53.

28. Grimshaw JM, Eccles MP, Lavis JN, Hill SJ, Squires JE. Knowledge translation of research findings. Implement Sci. 2012;7.

29. Morris ZS, Wooding S, Grant J. The answer is 17 years, what is the question: understanding time lags in translational research. J R Soc Med. 2011;104(12):510-20.

\section{Supplementary Files}

This is a list of supplementary files associated with this preprint. Click to download.

- LIVEProtocolPRISMAP.docx 